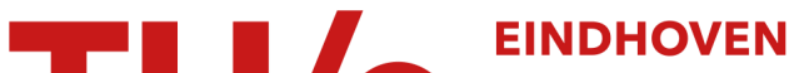 UNIVERSITY OF TECHNOLOGY
}

\section{Speed gradient control of chaotic continuous-time systems}

Citation for published version (APA):

Fradkov, A. L., \& Pogromski, A. Y. (1996). Speed gradient control of chaotic continuous-time systems. IEEE Transactions on Circuits and Systems I: Regular Papers, 43(11), 907-914. https://doi.org/10.1109/81.542281

DOI:

10.1109/81.542281

Document status and date:

Published: 01/01/1996

\section{Document Version:}

Publisher's PDF, also known as Version of Record (includes final page, issue and volume numbers)

\section{Please check the document version of this publication:}

- A submitted manuscript is the version of the article upon submission and before peer-review. There can be important differences between the submitted version and the official published version of record. People interested in the research are advised to contact the author for the final version of the publication, or visit the $\mathrm{DOI}$ to the publisher's website.

- The final author version and the galley proof are versions of the publication after peer review.

- The final published version features the final layout of the paper including the volume, issue and page numbers.

Link to publication

\section{General rights}

Copyright and moral rights for the publications made accessible in the public portal are retained by the authors and/or other copyright owners and it is a condition of accessing publications that users recognise and abide by the legal requirements associated with these rights.

- Users may download and print one copy of any publication from the public portal for the purpose of private study or research.

- You may not further distribute the material or use it for any profit-making activity or commercial gain

- You may freely distribute the URL identifying the publication in the public portal.

If the publication is distributed under the terms of Article 25fa of the Dutch Copyright Act, indicated by the "Taverne" license above, please follow below link for the End User Agreement:

www.tue.nl/taverne

Take down policy

If you believe that this document breaches copyright please contact us at:

openaccess@tue.nl

providing details and we will investigate your claim. 


\title{
Speed Gradient Control of Chaotic Continuous-Time Systems
}

\author{
Alexander L. Fradkov, Senior Member, IEEE, and Alexander Yu. Pogromsky
}

\begin{abstract}
The problems of synchronization and control of chaotic systems with uncertain parameters are considered as those of nonlinear adaptive control. To solve these problems for continuous-time systems the so called speed-gradient method is applied. As an example the problem of master-slave synchronization of the two forced Duffing's systems is considered.
\end{abstract}

\section{INTRODUCTION}

$\mathbf{R}$ ECENTLY THERE HAS BEEN some increasing interest in control of chaotic systems motivated by potential applications to the various fields of science and technology [1]-[9]. Different approaches including open-loop [4] and feedback [5] controls have been successfully applied (see surveys by Ogorzalek [6] and Chen and Dong [7]). The main advantage of the open-loop method is that no feedback signal is required. However the good model of the system is necessary and large parameter mismatch is not acceptable. On the opposite feedback methods allow to obtain good control results in the case of parameter uncertainty.

As an example of the controlled chaotic system the Duffing equation was studied in [5], [8], and [9]. In [8] and [9] it was examined how periodic parametric perturbations of small magnitude can destroy chaotic motion in the Duffing's system. This approach can also be related as open-loop and its applicability is based on the Melnikov theorem.

Closed-loop (feedback) control of the Duffing's system was proposed by Chen and Dong [5] who presented algorithms based on linear and nonlinear feedback and established conditions for local and global stability. Among the papers concerning feedback control of chaotic continuous-time systems we should also mention traditional PID approach [10], methods based on Lyapunov functions [11]-[13], high-gain feedback [14], feedback linearization (geometric approach) [15] and bifurcation control [16]. Some attempts have been also made to use concept of adaptive control of chaotic systems [17]-[19] where conditions of applicability were established by the local considerations i.e., by the linearization about equilibrium.

General approaches to synchronization and control of oscillatory systems based on Lyapunov functions method are suggested in [20]-[22]. In the present paper we further extend the ideas of [22] and give more detailed study of adaptive synchronization of two Duffing's systems. Our approach is

Manuscript received June 6, 1994; revised February 28, 1995 and November 15, 1995. This paper was recommended by Associate Editor M. Ogorzalek. The authors are with the Institute for Problems of Mechanical Engineering, St. Petersburg, 199178, Russia.

Publisher Item Identifier S 1057-7122(96)07599-X. based on the speed-gradient (SG) methodology that has been already successfully applied to design of various nonlinear and adaptive control systems [23]-[25]. The helpful feature of the SG method is that it makes possible to obtain the global stability conditions for overall adaptive system.

The paper is organized as follows. In Section II we pose the problem of adaptive control. Section III gives brief exposition of the SG method of adaptive control design. Section IV deals with the problem of adaptive synchronization of two Duffing's systems and numerical results are discussed in the Section V.

\section{Statement of the Adaptive Control Problem}

In this section we formulate the general nonlinear adaptive control problem as it has been stated in [23] and [24].

Suppose that the dynamics of the controlled system depends on the vector of unknown parameters. The essence of the adaptive control problem is to find such a control that the prespecified goal is achieved for all possible values of unknown parameters. In what follows we consider systems with finite state dimension, finite number of unknown parameters, with the control goal introduced by an objective functional.

Definition 1 (Adaptive Control Problem): Given the plant equations

$$
\begin{aligned}
& \dot{x}=G(x, u, t, \xi), \\
& y=H(x, u, t, \xi), \quad t \geq 0
\end{aligned}
$$

where $x \in R^{n}, u \in R^{m}$ and $y \in R^{l}$ are the plant states, inputs and outputs, respectively; $\xi \in R^{p}$ is a vector of unknown parameters.

Given the control objective

$$
Q_{t} \rightarrow 0, \quad \text { when } t \rightarrow \infty
$$

where $Q_{t}=Q_{t}[x(s), u(s), \xi ; 0 \leq s \leq t]$ is the objective functional. Find the two-level control algorithm

$$
\begin{aligned}
& u(t)=U_{t}[y(s), u(s), \theta(s) ; 0 \leq s<t], \\
& \theta(t)=\Theta_{t}[y(s), u(s), \theta(s) ; 0 \leq s<t],
\end{aligned}
$$

such that the overall system (1), (3) meets the aim (2). Here $\theta(t) \in R^{k}$ is a vector of adjustable parameters.

The design of adaptive control law can be accomplished in two steps. First of all the main control law is designed under the assumption that all the parameters of the dynamic system are known. Obviously this "ideal" control law depends on unknown parameters. After that all unknown parameters of the "ideal" control law are replaced by the adjustable parameters 
and at the second step we find the adaptation law meeting the control goal. The first level of the algorithm (3a) forms the main loop of the system and is called the main control law. The second level (3b) is the adaptation law. Here we underline the difference between the system input $u$ and the vector of adjustable parameters $\theta$ : the control signal $u$ forms the main loop but the algorithm of tuning the vector $\theta$ forms the adaptation loop.

The problem posed above encompasses numerous particular problems of control and adaptation. Indeed, the choice

$$
Q_{t}=\left(x(t)-x_{*}\right)^{T} P\left(x(t)-x_{*}\right)
$$

where $x_{*}=$ const, $P^{T}=P>0$ is any prespecified positive definite $n^{*} n$ matrix, corresponds to the problem of state stabilization. If $x_{*}$ in (4) is replaced by $x_{*}(t)$ then the objective functional

$$
Q_{t}=\left(x(t)-x_{*}(t)\right)^{T} P\left(x(t)-x_{*}(t)\right)
$$

specifies the tracking problem. Notice that in the important particular case the vectors $x_{*}(t)$ and $y_{*}(t)$ can satisfy some auxiliary system

$$
\dot{x}_{*}=F_{*}\left(x_{*}, t\right), \quad y_{*}=G_{*}\left(x_{*}, t\right) .
$$

This system is often referred to as a reference model in control literature [26] because it specifies the desired behavior of the controlled system. It can be shown that the goal (2) formulated for the functional

$$
Q_{t}=\left(y(t)-y_{*}(t)\right)^{T} P\left(y(t)-y_{*}(t)\right)
$$

where $P=P^{T}>0$ is any prespecified positive definite $l^{*} l$ matrix corresponds to the problem of output synchronization of two systems (1) and (6) as it is posed in [20]. In what follows we study the synchronization problem in this framework.

\section{SPEED-GRADIENT METHOD}

Most of general methods in the nonlinear control theory so far are based on Lyapunov functions. It is well known that the problem of finding these functions is quite tricky. The useful feature of the SG method presented in this section is that it helps to find the Lyapunov function for overall system based on the objective function specifying the problem.

Suppose that the main control law (3a) has been already chosen, and $U_{t}[\cdots]$ is a memoryless operator. Then substitution of (3a) into (1a) leads to the following system with input vector $\theta$

$$
\dot{x}=F(x, \theta, t)
$$

(dependence on $\xi$ is omitted for brevity in this section). According to the problem posed in the previous section the control goal is

$$
Q_{t} \rightarrow 0, \quad \text { when } t \rightarrow \infty \text {. }
$$

We will consider the case when $Q_{t}$ is a local functional: $Q_{t}=Q(x(t), t)$, where $Q(x, t)$ is a scalar smooth nonnegative function. In this case one can determine scalar function $\omega(x, \theta, t)$ as the speed of change of $Q_{t}$ along the trajectory of system (7)

$$
\omega(x, \theta, t)=\frac{\partial Q(x, t)}{\partial t}+F^{T}(x, \theta, t) \nabla_{x} Q(x, t) .
$$

The idea of speed-gradient method is to change the input $\theta$ along the gradient of $\omega(x, \theta, t)$ in $\theta$. The most general, so called, combined form of the SG algorithm looks as follows: [24], [25]

$$
\frac{d}{d t}(\theta+\psi(x, t))=-\Gamma \nabla_{\theta} \omega(x, \theta, t)
$$

where $\psi(\cdot)$ is some vector function satisfying the pseudogradient condition

$$
\psi^{T} \nabla_{\theta} \omega(x, \theta, t) \geq 0
$$

and $\Gamma=\Gamma^{T} \geq 0$ is a $k \times k$ positive definite gain matrix. Equation (9) can also be rewritten in the finite-integral form

$$
\theta=-\psi(x, t)-\Gamma \int_{0}^{t} \nabla_{\theta} \omega(x, \theta, s) d s .
$$

It is also possible to construct $S G$ algorithm in the purely finite form (see [23])

$$
\theta=-\psi(x, t)
$$

General stability theorem for combined SG law (9) is formulated below

Theorem 1: Consider the systems (7), (9) under the following assumptions:

A1) $F(x, \theta, t)$ and $\nabla_{\theta} \omega(x, \theta, t)$ are bounded together with their partial derivatives on any bounded set of states $(x, \theta)$ of the system (7), (9) iniformly in $t \geq 0$ (regularity condition).

A2) $\omega(x, \theta, t)$ is convex in $\theta$ (convexity condition).

A3) There exist $\theta_{*} \in R^{k}$ and scalar uniformly continuous in each bounded region function $\rho(x) \geq 0, \rho(0)=0$ such that inequality $\omega\left(x, \theta_{*}, t\right) \leq-\rho(x)$ holds for all $x \in R^{n}$ (achievability condition).

A4) If $Q(x(t), t)$ is bounded then $x(t)$ is bounded as well (boundedness condition).

Then each trajectory $(x(t), \theta(t))$ of system (7), (9), (10) is bounded and $\lim _{t \rightarrow \infty} \rho(x(t))=0$.

Proof: The proof is presented in the Appendix.

Now we briefly explain the meaning of the assumptions. First assumption is standard regularity condition ensuring that the overall system (7), (9) has a solution $x(t), \theta(t)$ on some time interval. Assumption A3 means that there exists an "ideal" constant value of input $\theta_{*}$ ensuring the objective (for adaptive control problem $\theta_{*}$ is a vector of adjustable parameters of the controller). It means additionaly that objective function $Q$ is Lyapunov function of "ideal" system. Condition A4 is necessary to establish the global properties of the overall system.

To apply the Theorem the conditions A1-A4 should be checked to ensure that all the trajectories of the system satisfy $\lim _{t \rightarrow \infty} \rho(x(t))=0$. Then applying La Salle principle (or autonomous systems) or its extensions (see [27]) one can establish convergence of the trajectories to the set $\Omega_{0} \subset \Omega$ 
consisting of the whole trajectories of (7), (9) belonging to $\Omega=\{x: \rho(x)=0\}$.

Remark 1: If the function $\rho(\cdot)$ explicitly depends only on $Q(x, t)$ (i.e., $\rho=\rho(Q)$, where $\rho(Q)>0$ for $Q>0$ ) then the goal (8) is achieved (see [24]).

Remark 2: Notice that even in the case when the goal (8) is achieved the above conditions are not sufficient to prove the convergence of the adaptation parameters $\theta$ to the "ideal" values $\theta_{*}$ (e.g., convergence of estimates of the unknown parameters to their true values $\xi$ ). Additional conditions ensuring this additional identification goal are established in [23], and in this case SG technique can be used to build parameter identifier of a dynamic system. The identification approach has been already applied to adaptive control design of chaotic continuous-time systems [28].

Remark 3: The Theorem can be extended to the case when function $\psi(\cdot)$ depends explicitly on $\theta$ (see also [24]). Similar results for purely finite algorithms are also available [24] under the strong pseudogradient condition

$$
\psi^{T} \nabla_{\theta} \omega(x, \theta, t) \geq \beta\left\|\nabla_{\theta} \omega(x, \theta, t)\right\|^{\delta}
$$

for some $\beta>0, \delta \geq 1$. They allow to establish that the standard variable structure control algorithms (e.g., relay algorithm [32] for stabilization of Lorenz system) are the special case of SG algorithms (see [22]).

Now when the applicability conditions of the SG method have been established we can formulate the following procedure of the adaptive control design:

Step 1: Formulate the control goal in the form (8). The objective functional $Q_{t}$ can be chosen for example as it is described in the Section II.

Step 2: Under the assumption that all parameters $\xi$ of the controlled system are known, find the main control law

$$
u=U(y, \xi, t)
$$

ensuring condition A3 which in turn ensures achievement of the goal (8).

Step 3: Introduce vector of adjustable parameters $\theta$ in the main control law instead of the unknown parameters

$$
u=U(y, \theta, t) .
$$

Step 4: Find the SG algorithm (7) and check other conditions of Theorem 1 .

Note that it is not necessary to make final choice of the objective functional at the Step 1. It may be modified or changed later on before Theorem 1 is applied. It may happen also that both main control law and adaptation law designed according to the above procedure depend on the whole state vector $x$ rather than on measurable output $y$. In this case standard observer design may be applied (the achievement of the control objective may be established at least for the case of "fast" observer by means of singular perturbation technique (see [29]).

More about the SG methodology can be found in [24]. Now consider example demonstrating application of the SG algorithm to adaptive control of chaotic systems.

\section{Control of Duffing's System}

Duffing equation is one of the simpliest systems that show chaotic behavior. It describes many different physical systems and its dynamics is studied in various publications [5], [8], [9], [30], [31]. In this section we will study the problem of control of the forced Duffing equation written in the following form

$$
\ddot{x}+p \dot{x}+p_{1} x+x^{3}=q \cos \omega t
$$

where $p>0, q$ is unknown parameter.

Standard Lyapunov argument shows that for $p>0$ all trajectories of (16) are uniformly bounded with respect to $t$. Using Melnikov theorem it can be shown [31] that when some parameters of the (16) are varied, the trajectories display changes of dynamic behavior. It was demonstrated in [5], that the behavior of the equation with $p=0.4, p_{1}=-1.1, \omega=$ 1.8 and $q$ varying in certain range is

$$
\begin{aligned}
& \text { chaotic (Fig. 1(a)) for } q=1.800, \\
& \text { periodic (Fig. 1(b)) for } q=0.620 \\
& \text { chaotic (Fig. 1(c)) for } q=2.100 \text {. }
\end{aligned}
$$

Consider the problem of controlling a chaotic trajectory of the Duffing equation to the trajectory of the reference model. We make the assumption that control can be introduced into system (16) by an additive signal in the right hand side of the system. By introducing $\dot{x}_{1}=x_{2}$, controlled system (4.1) can be rewritten as

$$
\left\{\begin{array}{l}
\dot{x}_{1}=x_{2} \\
\dot{x}_{2}=-p_{1} x_{1}-x_{1}^{3}-p x_{2}+q \cos \omega t+u
\end{array}\right.
$$

where $u$ is the control. The reference model has similar structure and differs from (17) only by the value of parameter $q$

$$
\left\{\begin{array}{l}
\dot{x}_{1 m}=x_{2 m} \\
\dot{x}_{2 m}=-p_{1} x_{1 m}-x_{1 m}^{3}-p x_{2 m}+q_{m} \cos \omega t
\end{array}\right.
$$

The problem is to design the adaptive control algorithm ensuring the goal

$\left\|x_{1}(t)-x_{1 m}(t)\right\| \rightarrow 0, \quad\left\|x_{2}(t)-x_{2 m}(t)\right\| \rightarrow 0, \quad$ as $t \rightarrow \infty$

This problem is just the problem of master-slave synchronization [20]. If the goal (19) is achieved then the dynamics of the controlled (slave or response) system coincides with the dynamics of the reference model (master or drive system). Therefore appropriate choice of the value of parameter $q_{m}$ allows to modify the behavior of the controlled system.

To solve the problem introduce the error equations subtracting (18) from (17)

$\left\{\dot{e}_{1}=e_{2}\right.$

$\left\{\begin{array}{l}\dot{e}_{1}=e_{2} \\ \dot{e}_{2}=-p_{1} e_{1}-e_{1}^{3}-p e_{2}+\left(q-q_{m}\right) \cos \omega t-3 x_{1} x_{1 m} e_{1}+u\end{array}\right.$

where $e_{1}=x_{1}-x_{1 m}$ and $e_{2}=x_{2}-x_{2 m}$. According to the design procedure of the previous section assume that the value of parameter $q$ is known. Then the main control law for system (20) that ensures the aim (19) can be chosen as follows:

$$
u=-K e_{1}+3 x_{1} x_{1 m} e_{1}+\left(q_{m}-q\right) \cos \omega t
$$

where $K$ is the proportional feedback gain. The law (21) in case $q=q_{m}$ was suggested in [5]. 


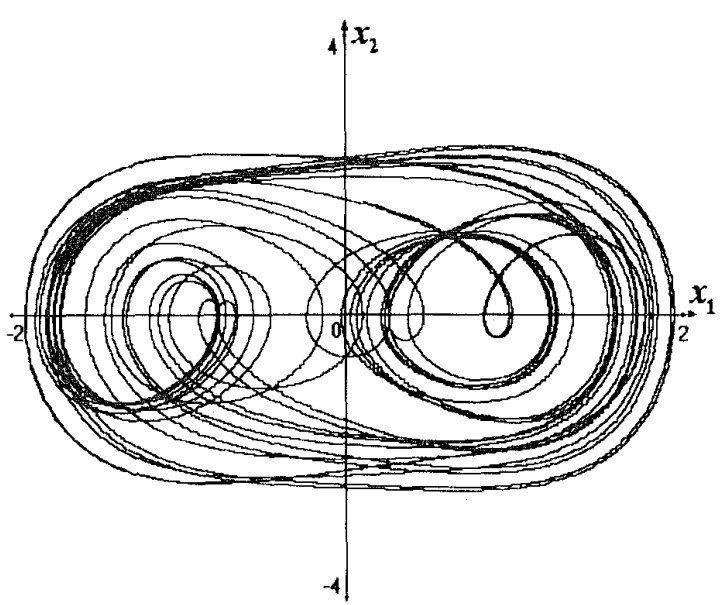

(a)

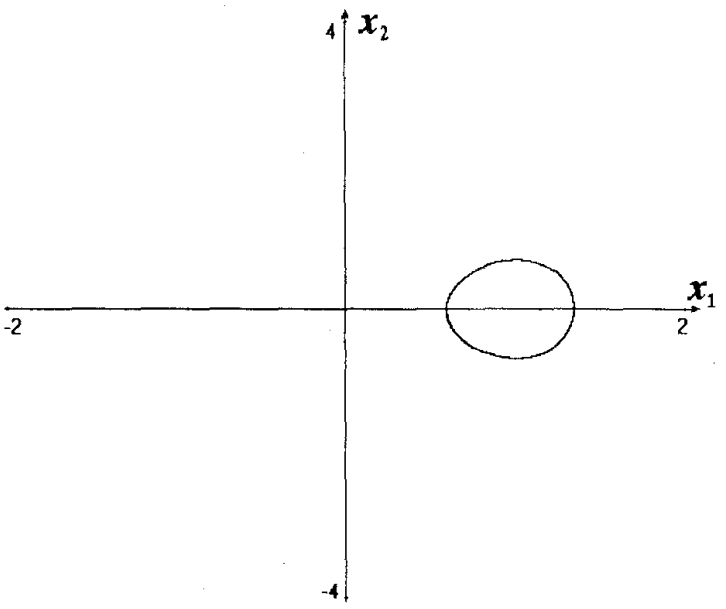

(b)

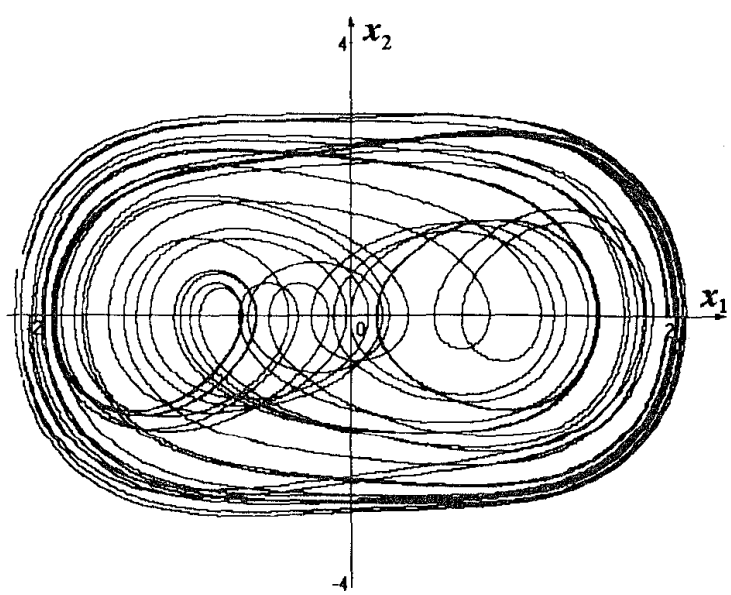

(c)

Fig. 1. Different solutions of Duffing's system: (a) $q=1.80$. (b) $q=0.62$. (c) $q=2.10$

The achievement of the synchronization goal (19) can be established by the following Lyapunov function [5]

$$
V(e)=\frac{1}{2}\left(\left(K+p_{1}\right) e_{1}^{2}+\frac{e_{1}^{4}}{2}+e_{2}^{2}\right)
$$

which is positive definite for $K>-p_{1}$. The derivative of (22) along (20) satisfies the inequality

$$
\begin{aligned}
\dot{V}(e)= & \left(K+p_{1}\right) e_{1} e_{2}+e_{1}^{3} e_{2}-p_{1} e_{1} e_{2} \\
& -e_{1}^{3} e_{2}-p e_{2}^{2}-K e_{1} e_{2} \\
= & -p e_{2}^{2} \leq 0 .
\end{aligned}
$$

It follows from (23) that the choice of function (22) as objective function ensures condition $\mathrm{A} 3$ with $\rho(e)=p e_{2}^{2}$, so we may choose $Q(e)=V(e)$.

Now recall that $q$ is unknown and pass to the Step 3 of the design procedure. Introduce the following main loop control law

$$
u=-K e_{1}+3 x_{1} x_{1 m} e_{1}+\theta \cos \omega t .
$$

According to the previous section we must calculate the "speed-gradient" of $Q(e)$ along the trajectories of the system. Direct calculations give

$$
\begin{aligned}
\dot{Q}(e)= & \left(K+p_{1}\right) e_{1} e_{2}+e_{1}^{3} e_{2}-p_{1} e_{1} e_{2}-e_{1}^{3} e_{2}-p e_{2}^{2} \\
& -K e_{1} e_{2}+e_{2}\left(q+\theta-q_{m}\right) \cos \omega t \\
= & e_{2}\left(q+\theta-q_{m}\right) \cos \omega t-p e_{2}^{2}
\end{aligned}
$$

and

$$
\nabla_{\theta} \dot{Q}=\frac{\partial Q}{\partial \theta}=e_{2} \cos \omega t
$$

Choosing pseudogradient function $\psi(e, t)=\lambda \nabla_{\theta} \dot{Q}=$ $\lambda e_{2} \cos \omega t$ one can obtain the SG adaptation algorithm in the combined form

$$
\theta=-\lambda e_{2} \cos \omega t-\gamma \int_{0}^{t} e_{2} \cos (\omega s) d s
$$

where $\lambda, \gamma>0$ are the adaptation gains.

To analyze the global behavior of the adaptive system with the main control law (24) and the adaptation law (27) check the conditions of Theorem 1: Conditions A1, A2 are valid since the right-hand sides of (20), (24) and (27) are smooth and linear in $\theta$. Condition A3 is valid since for $\theta_{*}=q_{m}-q$ we have $\dot{Q}(e)=-\rho(e)$, where $\rho(e)=p e_{2}^{2} \geq 0$. The validity of condition $\mathrm{A} 4$ follows from the radial unboundedness of the objective function (22) and boundedness of the trajectories of the reference model (18). Since all conditions of Theorem 1 are satisfied we conclude that all the trajectories of the system (18), (20), (24), (27) are bounded and $\rho(e(t))=p e_{2}^{2}(t) \rightarrow 0$. Since $\dot{e}_{2}=\ddot{e}_{1}$ is bounded, it yields that $e_{1}(t) \rightarrow 0$ and the goal (19) is achieved. In other words we proved that the designed adaptive controller ensures synchronization of two Duffing's systems when parameter $q$ of the slave (controlled) system is unknown and differs from the corresponding parameter of the master system.

One can notice that the problem of adaptive synchronization has been solved in this section under the assumption that master and slave systems differ from each other only by one parameter $q$. The other parameters of the two systems are assumed to be equal. This assumption has been made just for simplicity and it is not a limitation of the SG method. Therefore we may design adaptive controller when all parameters of the slave (controlled) systems differ from the 
corresponding parameters of the master system. Moreover in this paper we made an assumption that periodic perturbations in the right hand sides of the master and slave systems are in phase. It is also possible to solve the problem of synchronization in case when cosine term in (17) contents the phase uncertainty. In this case one can modify (17) to read

$$
\left\{\begin{array}{l}
\dot{x}_{1}=x_{2} \\
\dot{x}_{2}=-p_{1} x_{1}-x_{1}^{3}-p x_{2}+q \cos (\omega t+\varphi)+u
\end{array}\right.
$$

where parameters $p_{1}, p, q, \varphi$ are unknown and the goal of control is to sinchronize (28) and the following reference model

$$
\left\{\begin{array}{l}
\dot{x}_{1 m}=x_{2 m} \\
\dot{x}_{2 m}=-p_{1 m} x_{1 m}-x_{1 m}^{3}-p_{m} x_{2 m}+q_{m} \cos \omega t
\end{array}\right.
$$

Taking the main control law

$u=\theta_{1} e_{1}+\theta_{2} x_{1 m}+\theta_{3} x_{2 m}+3 x_{1} x_{1 m} e_{1}+\theta_{4} \cos \omega t+\theta_{5} \sin \omega t$

with adjustable parameters $\theta_{i}, i=1 \cdots 5$ and $\mathrm{SG}$ adaptation laws

$$
\begin{aligned}
& \theta_{1}=-\lambda_{1} e_{1} e_{2}-\gamma_{1} \int_{0}^{t} e_{1}(s) e_{2}(s) d s \\
& \theta_{2}=-\lambda_{2} x_{1 m} e_{2}-\gamma_{2} \int_{0}^{t} x_{1 m}(s) e_{2}(s) d s \\
& \theta_{3}=-\lambda_{3} x_{2 m} e_{2}-\gamma_{3} \int_{0}^{t} x_{2 m}(s) e_{2}(s) d s \\
& \theta_{4}=-\lambda_{4} e_{2} \cos \omega t-\gamma_{4} \int_{0}^{t} e_{2}(s) \cos (\omega s) d s \\
& \theta_{5}=-\lambda_{5} e_{2} \sin \omega t-\gamma_{5} \int_{0}^{t} e_{2}(s) \sin (\omega s) d s, \\
& \lambda_{i} \geq 0, \gamma_{i}>0
\end{aligned}
$$

one can establish similarly to the considered example that the goal (19) is achieved.

It is worth noting that solved problem of adaptive synchronization of two Duffing's systems encompasses the particular problem of adaptive stabilization about zero equilibrium of the Duffing's system. To solve this problem one must put the initial conditions and parameter $q_{m}$ of the reference model equal to zero. Therefore in the control law (30) and adaptation law (31) the error variables $e_{1}$ and $e_{2}$ should be replaced $x_{2}$ by $x_{1}$ and, respectively, while the reference variables $x_{1 m}$ and $x_{2 m}$ should be taken zero.

The above algorithms are suitable to promote or eliminate chaotic behavior in the controlled system no matter what kind of behavior-chaotic or regular was observed in the original system before control was applied.

\section{Simulation Results}

In this section we present results of computer simulation of the adaptive syncronization of systems (17) and (18) with control algorithm (24), (27).

1) According to [5], the Duffing equation has a chaotic solution [Fig. 1(a)] with parameters $p=0.4, p_{1}=$ $-1.1, q=1.8, \omega=1.8$ and a globally stable periodic solution [Fig. 1(b)] with parameters $p=0.4, p_{1}=$ $-1.1, q=0.62, \omega=1.8$. We first want to control the Duffing's system with chaotic behavior or to synchronize it with the system with stable limit cycle.

Let $q$ and $q_{m}$ be equal to 1.8 and 0.62 , respectively, with other relevant parameters $p, p_{1}$, and $\omega$ in the proposed controller. This choice ensures that for arbitrary initial conditions the driven Duffing equation has a chaotic response before control is applied and the reference system has stable dynamics.

Fig. 2(a) and (b) show how proposed control affects the original Duffing equation. Fig. 2(a) represents evolution of the regulation error in space $\left(x_{1}-x_{1 m}, x_{2}-x_{2 m}\right)$. It is clear from the figure that regulation error vanishes as time increases. Fig. 2(b) shows the transient process in the adaptive system in the space $\left(x_{1}, x_{2}\right)$. Constants $\lambda, \gamma$, and $K$ have been chosen to be equal to 50,15 , and 4 , respectively. It may be seen that the controlled trajectory tends to the stable orbit which is the globally attracting solution of the reference model.

2) The second example illustrates the ability of the SG algorithm to drive a chaotic solution of the Duffing equation to another chaotic one. In this case we want to tune the original plant with chaotic behavior [Fig. 1(a)] such that its trajectory becomes close to another chaotic motion [Fig. 1(c)].

Like in the above considered case regulation error vanishes [Fig. 3(a)]. This plot illustrates the case with $q=1.8, q_{m}=$ 2.1, and $\lambda=50, \gamma=15, K=4$. Fig. 3(b) is similar to Fig. 3(a) but control gain has been decreased: $\lambda=0.3, \gamma=$ 0.1 . It can be seen that in this case closed loop system is more oscillatory.

Therefore Examples 1 and 2 demonstrate that proposed adaptive controller can drive chaotic trajectory of the Duffing's system to any solution of the reference Duffing equation in case of parameter uncertainty.

In each of the presented cases the control is applied when $t=0$ with the following initial conditions $x_{1}(0)=x_{1 m}(0)=$ $x_{2 m}(0)=0, x_{2}(0)=2$.

\section{CONCLUSION}

Proposed method can be applied for problems of adaptive control and allows to obtain conditions of the global stability for overall system..

Considered example demonstrates how to utilize the speedgradient method for adaptive synchronization of two dynamic systems: it has been shown how to design adaptive controller for Duffing equation ensuring the convergence of its trajectories to periodic or chaotic solution of the reference model also described by Duffing equation. This example shows that the the goal of synchronization is still achieved, while the amplitude of the exciting periodic force or other parameters are unknown. This distinguishes the suggested algorithm from the existing ones [5], [12], [13].

The SG method has been applied previously to various problems of adaptation and control. The results of both the present paper and related ones [21], [22], [28], [33], [34] show 


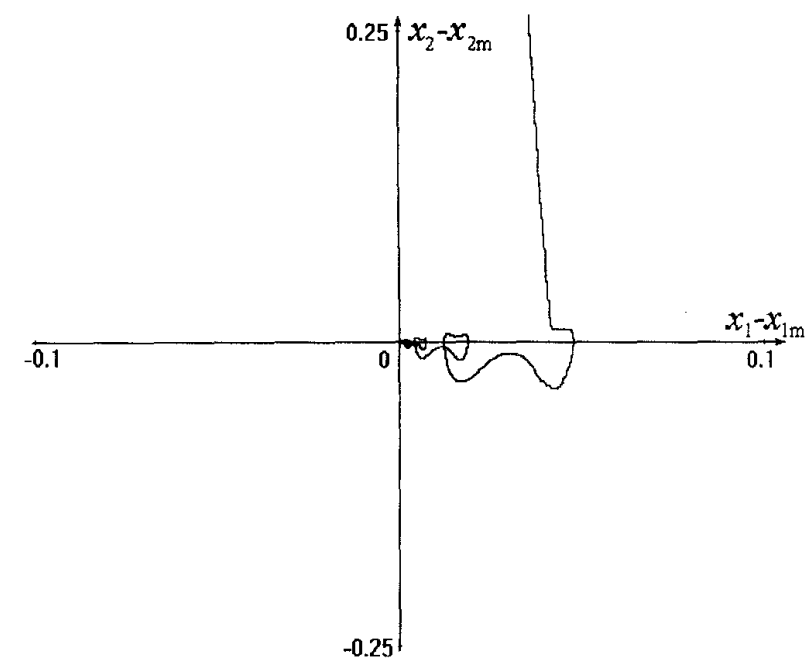

(a)

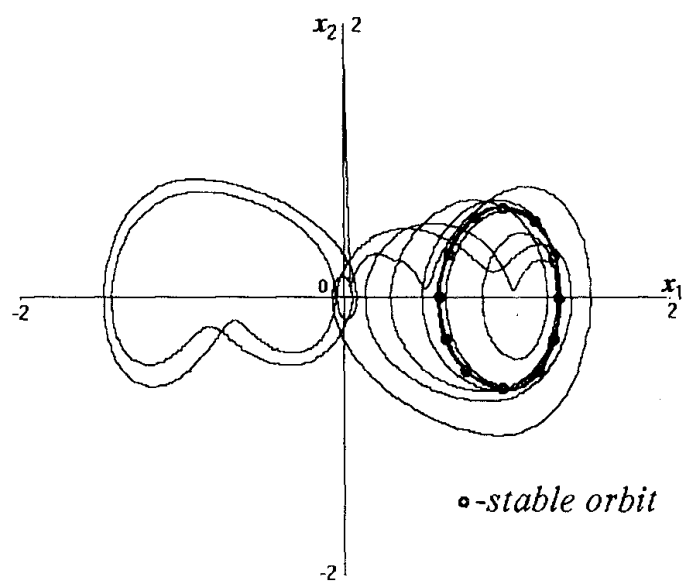

(b)

Fig. 2. Synchronization between two Duffing's systems. Reference trajectory is stable circle corresponding to $q=0.62$.

that it is a method of promise in synchronization and control of chaotic systems.

\section{APPENDIX}

Notice that condition A1 ensures that the Cauchy problem for system (7), (9) has the unique solution for arbitrary initial conditions $x(0), \theta(0)$ at least on some time interval.

Consider the following nonnegative Lyapunov function candidate

$$
\begin{aligned}
V(x, \theta, t)= & Q(x, t)+\frac{1}{2}\left(\theta_{*}-\theta-\psi(x, \theta, t)\right)^{T} \\
& \times \Gamma^{-1}\left(\theta_{*}-\theta-\psi(x, \theta, t)\right) .
\end{aligned}
$$

Its derivative along trajectories of (7), (9) satisfies

$$
\begin{aligned}
\dot{V}(x, \theta, t)= & \omega(x, \theta, t)+\left(\theta_{*}-\theta-\psi(x, \theta, t)\right)^{T} \nabla_{\theta} \omega(x, \theta, t) \\
= & \omega(x, \theta, t)+\left(\theta_{*}-\theta\right)^{T} \nabla_{\theta} \omega(x, \theta, t) \\
& -\psi^{T}(x, \theta, t) \nabla_{\theta} \omega(x, \theta, t)
\end{aligned}
$$

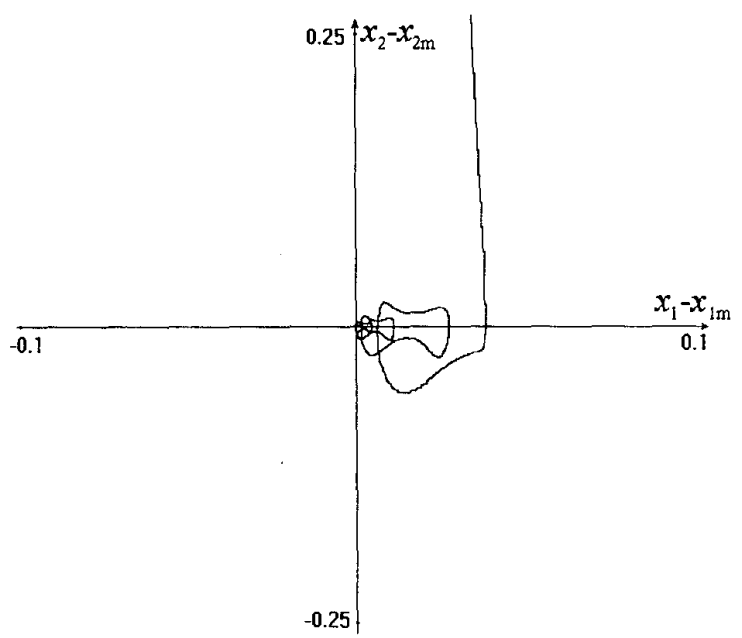

(a)

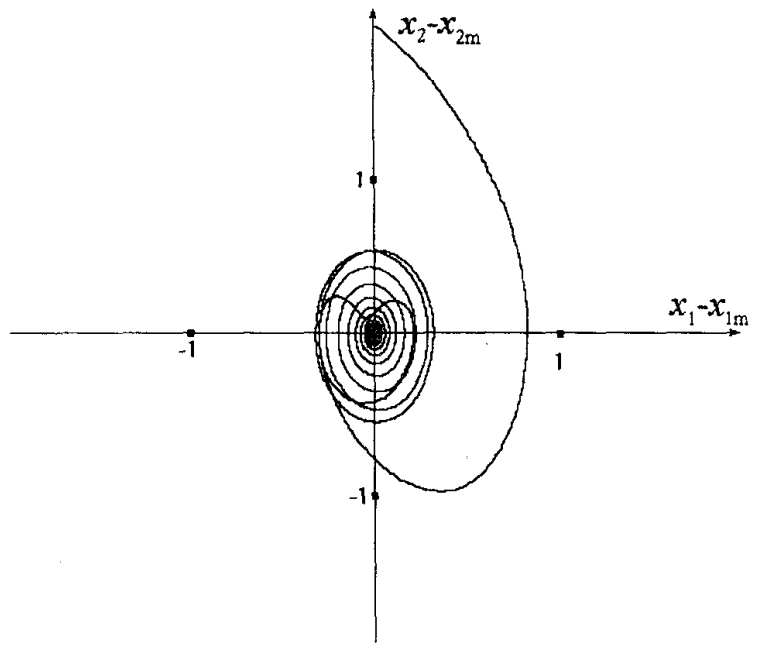

(b)

Fig. 3. Synchronization between two Duffing's systems. Reference trajectory is chaotic trajectory corresponding to $q=2.10$ : (a) $\lambda=50, \gamma=15$. (b) $\lambda=0.3, \gamma=0.1$.

Using pseudogradient property (10) and convexity conditions we get

$\dot{V}(x, \theta, t) \leq \omega(x, \theta, t)+\left(\theta_{*}-\theta\right)^{T} \nabla_{\theta} \omega(x, \theta, t) \leq \omega\left(x, \theta_{*}, t\right)$.

Achievability condition A3 gives

$$
\dot{V}(x, \theta, t) \leq-\rho(x) \leq 0
$$

i.e., $V(x, \theta, t) \leq V(x(0), \theta(0), 0)=V_{0}$, for any $t \geq 0$. Therefore $0 \leq Q(x(t), t) \leq V(x, \theta, t) \leq V_{0}$ and condition A4 yields existence and boundedness of trajectories $x(t), \theta(t)$ of the system (7), (9). for $t \geq 0$. Integrating (A.1) gives $\int_{0}^{\infty} \rho(x(t)) d t \leq V_{0}$ and taking into account uniform continuity of $\rho(x)$ on any bounded set and since $\rho(x) \geq 0$ by definition, one can conclude that $\lim _{t \rightarrow \infty} \rho(x(t))=0$ (see also [27]). It completes the proof of Theorem 1 . 


\section{REFERENCES}

[1] E. Hunt and G. Johnson, "Keeping chaos at bay," IEEE Spectrum, pp. 32-36, Nov. 1993.

[2] L. Kocarev, K. Halle, K. Eckert, and L. Chua, "Experimental demonstration of secure communications via chaos synchronization," Int. $J$ Bifurc. Chaos, vol. 2, pp. 709-713, 1992.

[3] E. Ott, C. Grebogi, and J. Yorke, "Controlling chaos," Phys. Rev. Lett., vol. 64 , pp. 1196-1199, 1990.

[4] A. Huebler and E. Luscher, "Resonant stimulation and control of nonlinear oscillators," Naturwissenschaften, vol. 76, pp. 67-69, 1989.

[5] G. Chen and X. Dong, "On feedback control of chaotic continuous-time systems," IEEE Trans. Circuits Syst., vol. 40, pp. 591-601, Sept. 1993.

[6] M. J. Ogorzalek, "Taming chaos," IEEE Trans. Circuits Syst., vol. 40, pp. 693-706, Oct. 1993.

[7] G. Chen and X. Dong, "From chaos to order-perspectives and methodologies in controlling chaotic nonlinear dynamic systems," Int J. Bifurc. Chaos, vol. 3, pp. 1363-1409, 1993.

[8] R. Lima and M. Pettini, "Suppression of chaos by resonant parametric perturbations," Phys. Rev. A, vol. 41, pp. 726-733, 1990.

[9] L. Fronzoni, M. Geocondo, and M. Pettini, "Experimental evidence of suppression of chaos by resonant parametric perturbations," Phys. Rev. A, vol. 43, pp. 6483-6487, 1991.

[10] T. Hartley and F. Mossayebi, "A classical approach to controlling the Lorenz equations," Int. J. Bifurc. Chaos, vol. 2, pp. 881-887, 1992

[11] Y. Chen and M. Chou, "Continuous feedback approach for controlling chaos," Phys. Rev. E, vol. 50, pp. 2331-2334, 1994.

[12] H. Nijmeijer and H. Berghuis, "On Lyapunov control of Duffing equation," IEEE Trans. Circuits Syst., vol. 42, pp. 473-477, 1995.

[13] K. S. Tang, K. F. Man, and S. Kwong, "Nonlinear dissipative controller for chaotic system," in IFAC Symp. Nonlinear Contr. Syst. (NOLCOS'95), pp. 789-794.

[14] L. Kocarev, A. Shung, and L. O. Chua, "Transition in dynamic regime by driving: A method of control and synchronization of chaos.," Int. J. Bifurc. Chaos, vol. 3, pp. 479-483, 1993.

[15] J. Alvarez-Gallegos, "Nonlinear regulation of a Lorenz system by feedback linearization techniques," Dynamics and Control, vol. 4, pp. 277-298, 1994

[16] H. Wang and E. H. Abed, "Bifurcation control of chaotic dynamic system," IFAC Symp. NOLCOS'93, pp. 283-288.

[17] B. Huberman and E. Lumer, "Dynamics of adaptive systems," IEEE Trans. Circuits Syst, vol. 37, pp. 547-550, 1990.

[18] S. Sinha and R. Ramaswamy, "Adaptive control in nonlinear dynamics," Physica D, vol. 43, pp. 118-128, 1990.

[19] D. Vassiliadis, "Parametric adaptive control and parameter identification of low-dimensional chaotic systems," Physica D, vol. 71, pp. 319-341, 1994.

[20] C. Wu and L. Chua, "A unified framework for synchronization and control of dynamical systems," Int. J. Bifurc. Chaos, vol. 4, pp. 979-998, 1994.

[21] A. Fradkov, "Nonlinear adaptive control: Regulation-trackingoscillations," in Ist IFAC Workshop on New Trends in Control Systems Design, Smolenice, 1994, pp. 426-431.

[22] A. L. Fradkov and A. Yu. Pogromsky, "Speed gradient adaptive control of chaotic continuous-time systems," in Preprint 107 Saint Petersburg: Inst. Probl. Mech. Eng. (IPME), 1994, pp. 29-47.

[23] V. N. Fomin, A. L. Fradkov, and V. A. Yakubovich, Adaptive Control of Dynamic Objects. Moscow: Nauka, 1981, (in Russian).

[24] A. L. Fradkov, Adaptive Control in Large-Scale Systems. Moscow: Nauka, 1990, (in Russian)

[25] B. R. Andrievsky, A. A. Stotsky, and A. L. Fradkov, "Speed-gradient algorithms in control and adaptation. A survey." Automat. Remote Contr., vol. 49, Part 1, pp. 1533-1564, 1988

[26] L. Landau, "A survey of model reference adaptive techniques-Theory and Applications," Automatica, vol. 10, pp. 353-379, 1974.

[27] C. I. Byrnes and C. F. Martin, "An integral-invariance principle for nonlinear systems," IEEE Trans. Automatic Contr., vol, 40, pp. 983-994, June 1995.
[28] A. Pogromsky, "Identification based adaptive control of chaotic continuous-time systems," in IFAC Conf. Syst., Structures Contr. Nantes, 1995, pp. 629-634.

[29] A. L. Fradkov, "Synthesis of adaptive control systems for nonlinear singularly perturbed plants," Automat. Remote Contr., vol. 48, pp. 789-798, 1986.

[30] F. Moon, Chaotic Vibrations. New York: Wiley, 1987.

[31] J. Guckenheimer and P. Holmes, Nonlinear Oscillations, Dynamical Systems, and Bifurcations of Vector Fields. New York: Springer-Verlag, 1983.

[32] J. Singer, Y.-Z. Wang, and H. Bau, "Controlling a chaotic system," Phys. Rev. Lett., vol, 66, pp. 1123-1125.

[33] A. L. Fradkov, A. Yu. Pogromsky, and A. Yu. Markov, "Adaptive control of chaotic continuous-time systems," in Proc. 3rd Euro. Contr. Conf. (ECC'95), pp. 3062-3067.

[34] A. L. Fradkov, P. Yu. Guzenko, D. J. Hill, and A. Yu. Pogromsky, "Speed gradient and passivity of nonlinear oscillators," in IFAC Symp. Nonlinear Contr. Syst. (NOLCOS'95), pp. 655-659.

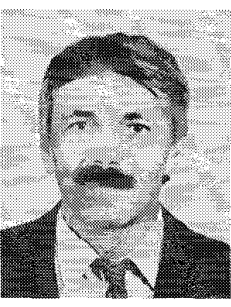

Alexander L. Fradkov (SM' 95) was born on May 22,1948 . He graduated from the mathematicalmechanical faculty of St. Petersburg State University in 1971. He received the Ph.D. degree in 1975 and the D.Sc. degree in 1986 from St. Petersburg Electrotechnical Institute.

From 1971 to 1987 he occupied different research positions with Baltic State Technical University (BSTU). Then, in 1987 became Professor of computer science at BSTU. Since 1990 he has been the Head of the Laboratory of Complex Systems Control of the Institute for the Problems of Mechanical Engineering and a part time professor with the Control Systems Department of BSTU. His current interests are in fields of nonlinear adaptive control, control of oscillatory and chaotic systems and computer-aided control systems design. He has published seven books and textbooks, among them are Applied Theory of Discrete Adaptive Control Systems (with D. P. Derevitsky, Nauka, 1981), Adaptive Control of Dynamic Systems (with V. N. Fomin and V. A. Yakubovich, Nauka, 1981), Adaptive Control of Complex Systems (Nauka, 1990) and approximately 200 journal and conference papers. He is coauthor of more than 50 technical reports. During 1991-1995 he visited and gave lectures in more than 40 universities of 12 countries.

Dr. Fradkov is the Vice-President of the St. Petersburg Informatics and Control Society, a Member of the Board of the Russian Scientific Society of Control Systems and Processes; a member of SIAM and the New York Academy of Sciences. He was co-chairman of the International Student Olympiades on Automatic Control in 1991-1996; a member of the Young Author Prize Committee at the 12th IFAC World Congress (Sydney, 1993). $\mathrm{He}$ is a member of the IEEE CSS "Engineers at Risk" Committee and a Member of the International Program Committees of European and Asian Control Conferences of 1997.

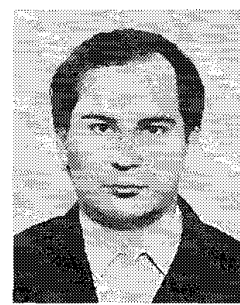

Alexander Yu. Pogromsky was born in 1970. He received the M.S. degree in electrical engineering from Baltic State Technical University in 1991 and the $\mathrm{Ph} . \mathrm{D}$. degree, also in electrical engineering, from St. Petersburg Electrotechnical University in 1994.

In 1994 he joined the Laboratory of Complex Systems Control in the Institute for the Problems of Mechanical Engineering. His current fields of scientific interests are nonlinear control, adaptive control, and electromechanical systems. 